\title{
The essence of light
}

This paper was downloaded from TechRxiv (https://www.techrxiv.org).

\section{LICENSE}

CC BY 4.0

SUBMISSION DATE / POSTED DATE

03-10-2021 / 06-10-2021

CITATION

Zheng shengming, Physicist (2021): The essence of light. TechRxiv. Preprint. https://doi.org/10.36227/techrxiv.16727020.v1

$\mathrm{DOI}$

10.36227/techrxiv.16727020.v1 


\section{The essence of light}

Zheng shengming (郑胜明)（Physicsworks@tom.com）

The contents of this article fall within Fields, Wave and Electromagnetics; Photonics and Electrooptics area of scope of TechRxiv, part of it are published in my book (ISBN: 9789881767592) but is not published in journal. For convenience readers, I revise and submit it to here

Abstract: In the process of exploring the essence of light, Newton initially agreed with the particle interpretation of light while Huygens argued for the wave theory. Hence, these two theories had been disputed in Newton's time. In the beginning people accepted the particle theory, but after Thomas Young's experiment and Augustin Jean Fresnel's experiment, people began to accept the wave theory. Until Einstein proposed the quanta concept, which was later called photon, and, even later, De Broglie proposed the wave nature of matter, subsequently, people began using particle-wave duality to explicate all phenomena in micro world. Thus here appears a paradox: how can one particle exist in two forms? To solve this enigma, I have done some experiments; discover that moving photons create force; this effect reveal the phenomenon of light wave property-the inference fringes is caused by force which moving photons produced. The essence of light is particle but not particle-wave duality.

\section{Chapter.1. The interference fringe is produced by Force}

One famous phenomenon that changing from dark to bright in the diffractive center has always been used to verify the wave theory of light. This seems to demonstrate the existence of light wave. The below experiment shows that the center of light beam changing from dark to bright is not an effect of periodic wave, but is the result of force interaction. As in Figure1, a light source is created, it emits two light beams, like in picture a of Figure1. They do not contact each other in their moving process while there is a $1.5 \mathrm{~mm}$ distance between them. Their tracks of moving are shown in picture $\mathbf{b}$ of Figure1. This experiment indicates when both light beams $\mathrm{O}$ and $\mathrm{P}$ move forward, the center of light beam $\mathrm{O}$ is dark on the screen, as shown in picture $\mathbf{c}$ of the Figure1; when only light beam $\mathrm{O}$ moves forward, the center of light beam $\mathrm{O}$ is bright on the screen. (See picture $\mathbf{d}$ in the Figure1) In this changing process, the radius of light beam $\mathrm{O}$ is $3.5 \mathrm{~mm}$, and there is $1.5 \mathrm{~mm}$ distance between light beam
$\mathrm{O}$ and light beam $\mathrm{P}$. This shows that the changing of light bean $\mathrm{O}$ has nothing to do with the interaction of contact force, because the light beam $P$ whether or not exists, the center of light beam $\mathrm{O}$ is far away from the edge of light beam $\mathrm{P}$, and they do not contact; when light beam $\mathrm{P}$ is removed, this action does not act on the light beam $O$. If the changing of the center of light beam from dark to bright is the result of periodicity of wave, the change in the center of light beam $\mathrm{O}$ would be same as the light beam traveled in an equal distance in these two states. But in this experiment, the center of light beam $\mathrm{O}$ is bright when the light beam $\mathrm{P}$ does not exist and the center of light beam $O$ is dark when the light beam $\mathrm{P}$ exists. The light passed an equal distance in these two states. This unequivocal indicates that center of light beam $\mathrm{O}$ changing from dark to bright is not the periodicity of the wave; but is due to force which moving photons created.
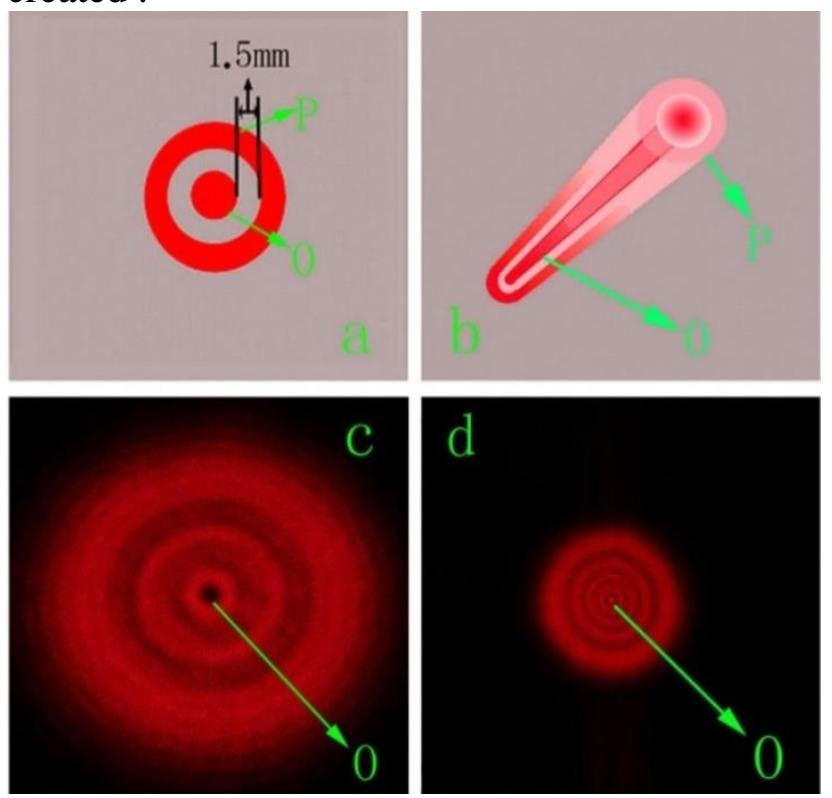

Figure1. Picture $\mathbf{a}$ is the cross-section of two light beams O, P. Picture $\mathbf{b}$ is the status of the moving track of light beams $\mathrm{O}, \mathrm{P}$. Picture $\mathbf{c}$ is the photograph in the screen when light beams $\mathrm{O}, \mathrm{P}$ are all moving forward; and picture $\mathbf{d}$ is the photograph in the screen when only light beam $\mathrm{O}$ moves forward. 
This experiment indicates that the center of light beam $\mathrm{O}$ diffraction changes from dark to bright is not a wave periodic change, but is the effect of the interaction of force which moving photons created.

In order to corroborate that the interference fringe of light is a result of interaction of force which moving photons created, experiment 2 is designed. (See Figure2) Three light beams A, O, and $\mathrm{C}$, are created. Their interaction also indicates that the wave phenomenon in light is produced by force. The detail sees below experiment 2.

Their interaction results are shown in the Figurer2. When outer part of the light beam A is removed, light beam $\mathrm{C}$ appear interference fringes and the left light beam A moves to light beam $\mathrm{C}$. This also bears out that the interference fringes of light, which shows the wave characteristics, are produced by the moving photons generate force.
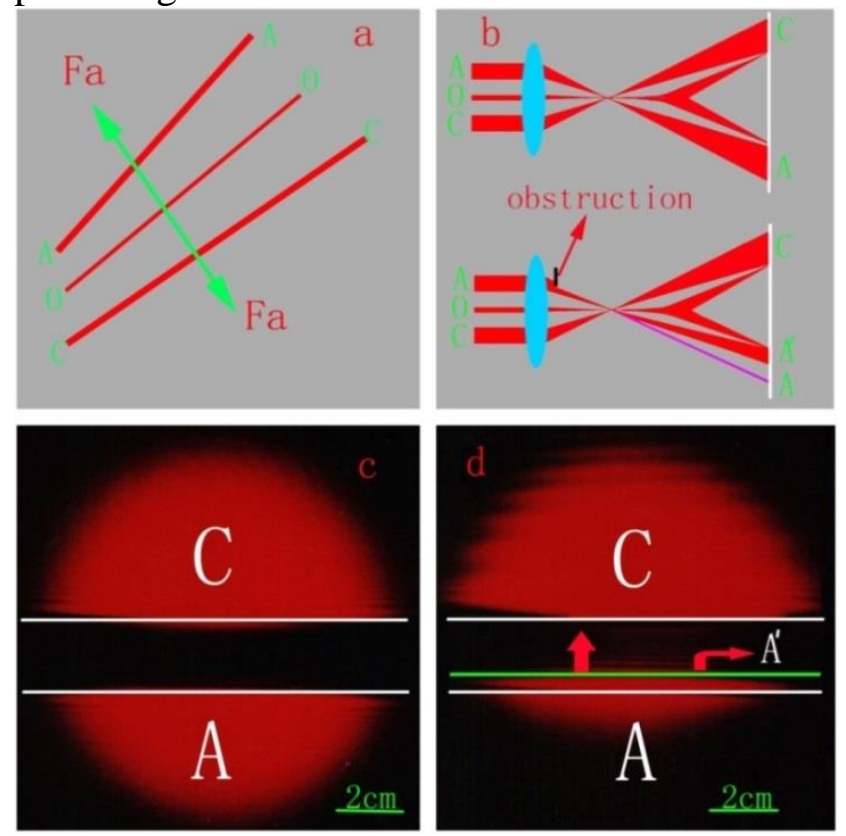

Figure2. Picture $\mathbf{a}$ is the status of force in the three light beams $\mathrm{A}, \mathrm{O}$, and $\mathrm{C}$; Picture $\mathbf{b}$ shows the motion state of light beams A, O, and C; Picture c is the photograph of light beams $\mathrm{A}$ and $\mathrm{C}$ when they are first projected on the screen; Picture $\mathbf{d}$ is the motion state when the outer part of light beam A has been removed. In picture $\mathbf{d}$, the white line is the original site of light beam A when outside light beam has not been removed; The green line is the site of light beam A when its outside part of light beam has been removed, and the red arrow is the direction of the light beam A accepting force; The purple line in picture $\mathbf{b}$ is the moving track of light beam A when its outer light beam has not been removed.

Further analysis of the above two experiments: we know that periodic wave is changed with two changing conditions: first it is changed with moving forward; secondly, when two waves contact they are changed by two wave's interaction. But in experiment 1 light beam $\mathrm{O}$ and light beam $\mathrm{P}$ did not contact in their traveled displacements and they passed distance are equal. According to wave theory, the change in the center of light beam $\mathrm{O}$ will be same, no matter it is dark or bright. But in fact, in this experiment, the change in the center of light beam $\mathrm{O}$ is not same even though traveled distance is equal. If the light beam $\mathrm{P}$ exists, the center of light beam $\mathrm{O}$ is dark; if the light beam $\mathrm{P}$ does not exist, the center of the light beam $O$ is bright. This cannot be elucidated by wave theory, but can be elucidated by above discovery. If the light beam $\mathrm{P}$ does not exist, light beam $\mathrm{O}$, accepted force from outside, will be lost; consequently, the centric light ray in light beam $\mathrm{O}$ does not move toward outside. In this condition it is bright. If light beam $\mathrm{P}$ exists, light beam $\mathrm{O}$ accepts outside force from light beam $P$, so the centric light beam moves to the outside, in this condition it is dark. In other words, the changing of light beam $\mathrm{P}$ affects the interaction force in light beam $O$. Because the moving photons bring on attraction of force, so vary light beam will change the distributing force in space.

In the picture a of Figure2, light beam $\mathrm{O}$ has been attracting by light beam $\mathrm{A}$ and light beam $\mathrm{C}$ in same time. Meanwhile, in light beam A, the inside part of light beam also accepts force from outside light beam; when outer parts of light beam A are removed, the inside light beam accepts force from outside light beam will be lost, light beam $\mathrm{O}$ accepts force from light beam A will be decreased. However, accepted force from light beam $\mathrm{C}$ does not change. Thus, light beam $\mathrm{O}$ and the left light beam A will move toward light beam C. Meanwhile light beam $\mathrm{C}$ accepted force from light beam $\mathrm{A}$ is also decreased. This will lead to change the original balance of force in the three light beams A,O,C; the original distribution of light beam $\mathrm{C}$ will be changed at this changing force status for new balance, in the end the light beam $\mathrm{C}$ become interference fringe like wave characteristics. These two experiments unequivocal demonstrate that the wave characteristic of light is brought on by the moving photons generate force, and it is indicated that the light is particle, namely the essence of light is particle, on the other 
hand, because moving photons create force, so changed light beam will lead to appear wave phenomenon when it moving forward at same time. This is the reason why the light seems wave-particle duality.

Chater.2. Applying in the light interference

\subsection{A method for describe the image of interference and diffraction:}

We now know that the light wave phenomenon is produced by force, which moving photons created. Based on this discovery, I came up with a new method to draw images of multi-pinhole diffraction patterns and their interference fringes. According to the above conclusion, the moving photons create force. Below, I do experiment 3 to apply this discovery. In picture a of Figure 3 when light beam goes through two pinholes $\mathrm{A}$ and $\mathrm{B}$, they will be closing as they disperse while moving forward. At their coinciding region $\mathrm{C}$, part of light beam $\mathrm{A}$ and part of light beam B can become a streak-line like $\mathbf{L}$, this is confirmed by the experiments in picture $\mathbf{b}$ of Figure 3. This becomes interference fringes between two pinholes; the picture $\mathbf{z}$ in Figure 3 confirms this conclusion. When two streaks intersect, the intensity of their intersected spot is higher than its surrounding region; hence the force at the spot of intersection is bigger than its outer region. This attracts the surrounding light rays to the center, and becomes a circle dot at their intersected point, as in picture $\mathrm{c}$ of Figure3.
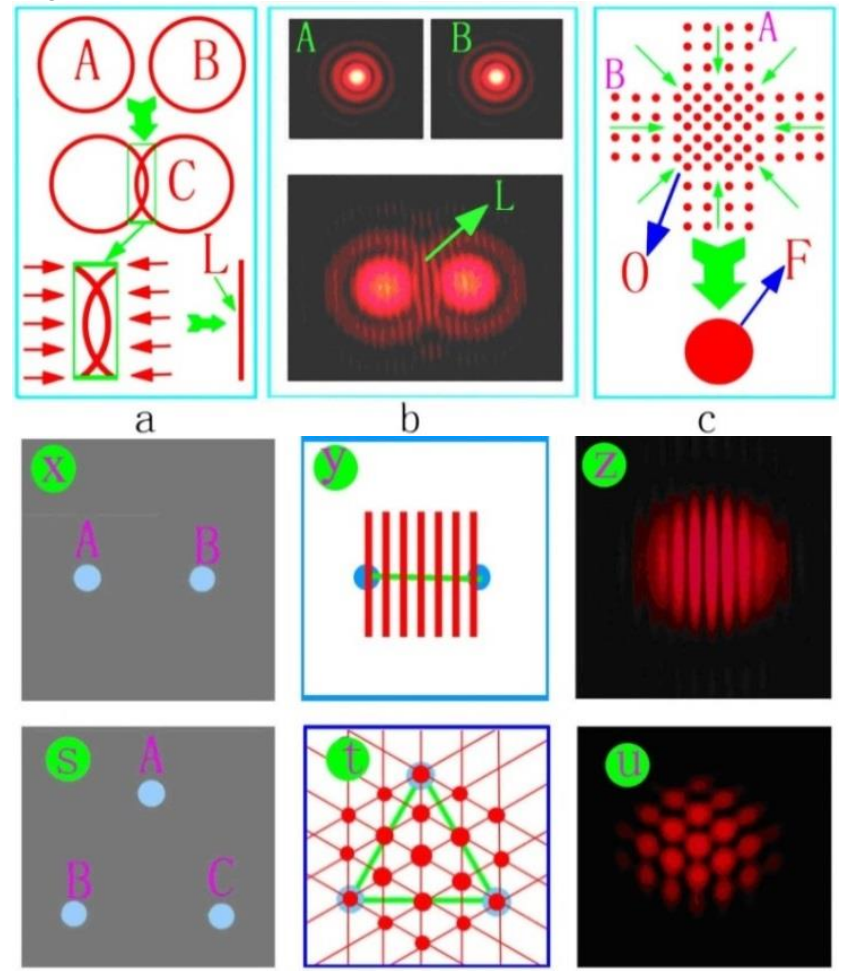
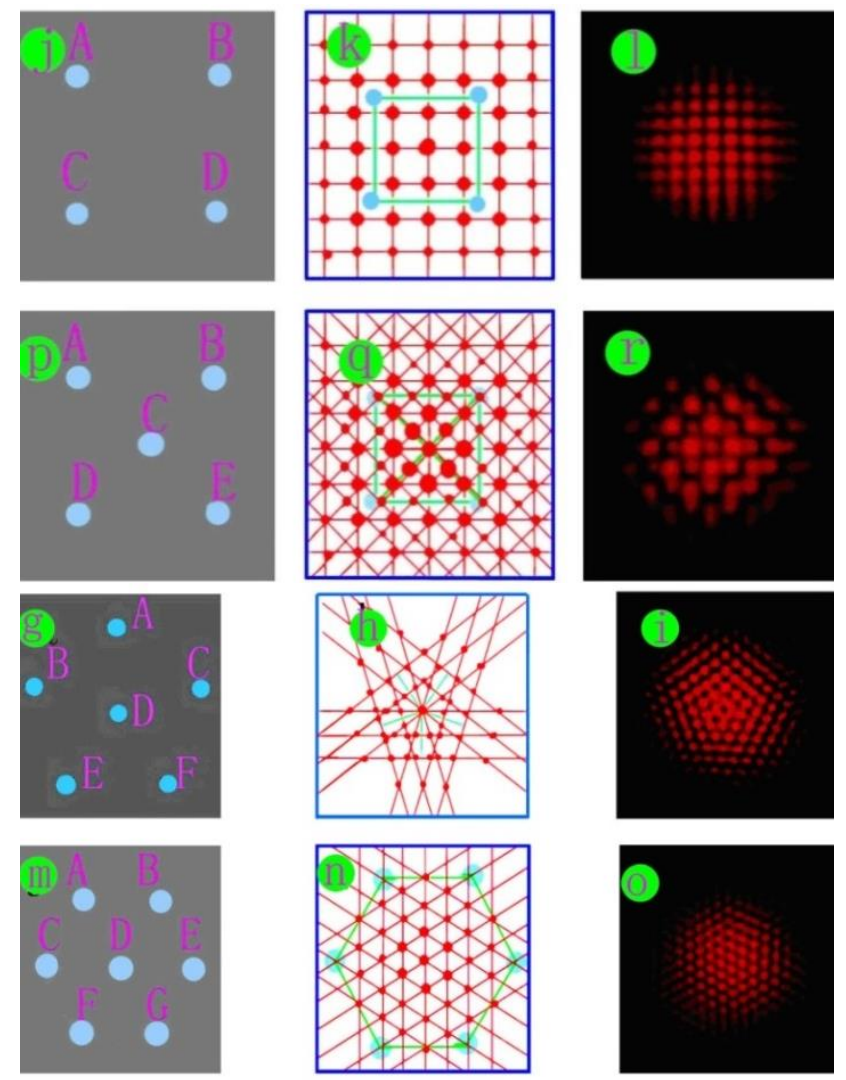

Figure3. a, b, c are the pictures that is indication of method; $\mathbf{x}, \mathbf{s}, \mathbf{g}, \mathbf{m}, \mathbf{j}, \mathbf{p}$ are pinhole lattices; $\mathbf{y}, \mathbf{t}, \mathbf{k}, \mathbf{q}, \mathbf{h}, \mathbf{n}$, are drawn pictures according to the conclusions of discovery; $\mathbf{z}, \mathbf{u}, \mathbf{l}, \mathbf{r}, \mathbf{i}, \mathbf{o}$, are photographs that light beam passing through pinholes lattice projected on the screen become.

Above meaning can be simply narrated below: First, the interference fringe became between two pinholes is perpendicular to the line which links two pinholes. Second, the intersecting spot of two streaks becomes a dot. According to this, I infer that when the light beam goes through three, four, five, or six pinholes, the image which light projects on the screen can be drawn below. Given the perpendicular line for the line which links the nearest two pinholes, the point of intersecting of this vertical line just right is the image become on the screen. There are two facts confirm this view. First, the intersected dot of the two streaks is in the original source of these two streaks. Second, the number of dots in the line is equal the number of streaks between two pinholes. In Figure 3, the pictures $\mathbf{x}, \mathbf{s}, \mathbf{g}, \mathbf{m}, \mathbf{j}, \mathbf{p}$ are the pictures of status of pinholes, and $\mathbf{z}, \mathbf{u}, \mathbf{l}$, $\mathbf{r}, \mathbf{i}, \mathbf{o}$ are photographs of light going through the 
pinholes projected on the screen. The pictures $\mathbf{y}$, $\mathbf{t}, \mathbf{k}, \mathbf{q}, \mathbf{h}, \mathbf{n}$ are drawn according to the above method. These pictures are in extremely good agreement with photographs $\mathbf{z}, \mathbf{u}, \mathbf{l}, \mathbf{r}, \mathbf{i}, \mathbf{o}$ that light goes through the pinholes become on the screen in the experiment. This experiment indicates that diffraction patterns and interference fringes projected on the screen can be drawn by according to the discovery that the moving photon creates force.

\subsection{Test the universality of above method}

The electron diffraction experiment further confirmation above method: In 1984 Shechtman, Blech, Gratias, Cahn took photograph of electron diffraction in $\mathrm{MnAl}$ alloy, they got the picture $\mathbf{b}$ in Figure 4 and picture $\mathbf{c}$ in Figure5. Now use above method can get a picture d, e, $\mathbf{f}$ in Figure4, and Figure5. Carefully comparison of took photograph and of draw picture to identify them as the same, namely they are completely match up with each other. That is to say this method is in extremely good agreement with experiment. For further compare them, carefully see the purple spot in the picture $\mathbf{b}, \mathbf{c}, \mathbf{e}, \mathbf{f}$ of Figure 5, their framework of geometry in space are completely identical!
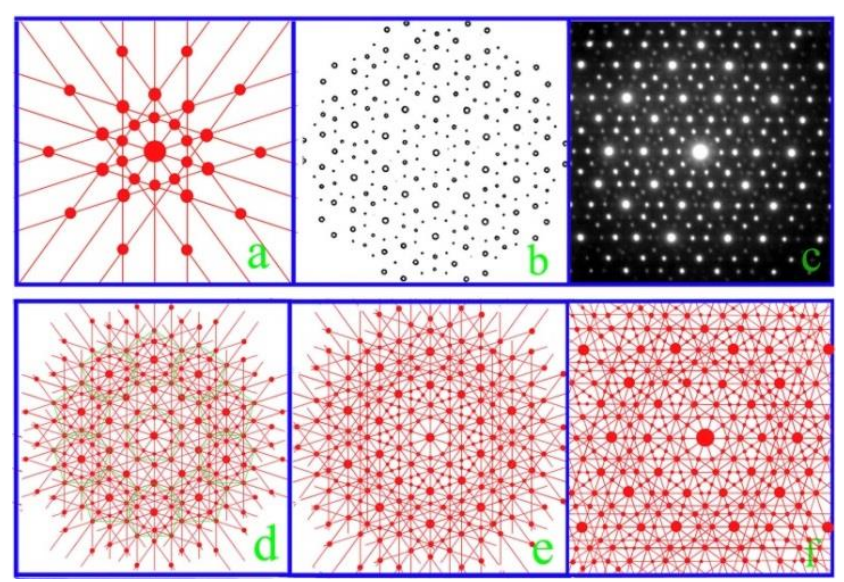

Figure4. Picture a show this method of draw diffraction graph. Picture $\mathbf{b}, \mathbf{c}$ are experimental photographs. Picture d, e, $\mathbf{f}$ are according to this method draw the diffraction graph.

From this we can see this method is completely validity. Namely it is in extremely good agreement with experiments.
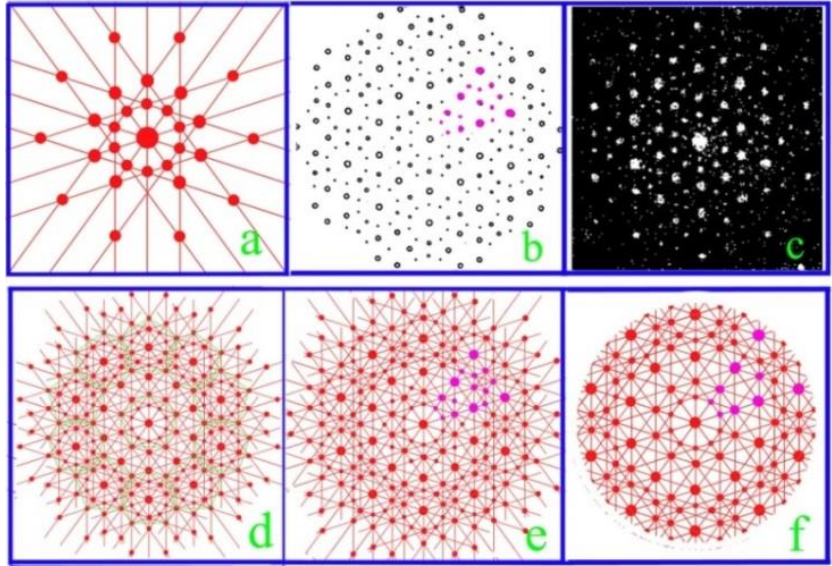

Figure5. Picture a is the method of draw diffraction image. Picture $\mathbf{b}, \mathbf{c}$ are experimental photographs. Picture d, e, $\mathbf{f}$ are according to this method draw the diffraction picture.

From compare the purple spot in the picture b, $\mathbf{c}$, e, f, it is indicating that their framework of geometry in space are completely identical!

For show the universality of above method, below we see another example: below is the photograph of electron diffraction in lizardite $\mathrm{Mg}_{3}\left(\mathrm{Si}_{2} \mathrm{O}_{5}(\mathrm{OH})_{4}\right.$. See below picture b in Figure7

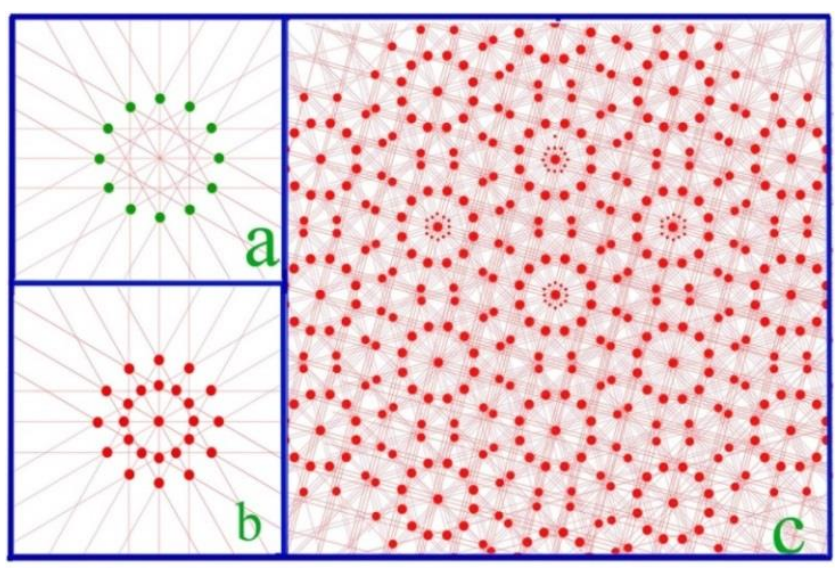

Figure6.Picture a, b show the method of draw photograph of electron diffraction in lizardite. Picture $\mathbf{c}$ is drawn by this method.

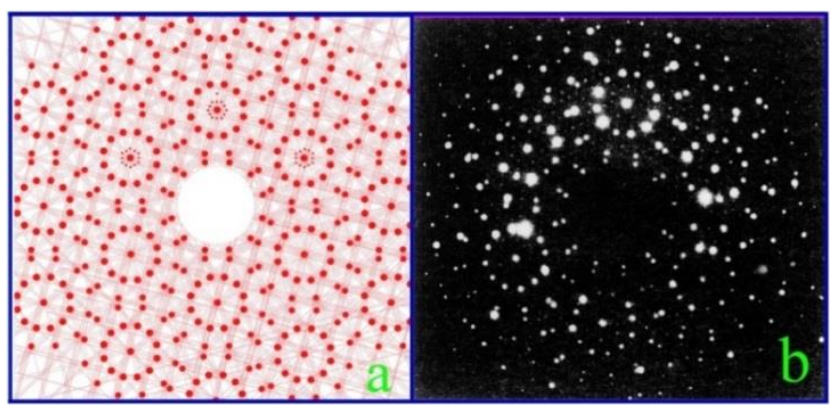

Figure7.Picture $\mathbf{b}$ is the photograph of 
experiment of electron diffraction in lizardite. Picture $\mathbf{a}$ is the picture that according my method draw.

\section{Chapter.3. Analysis Thomas young and Michelson experiment}
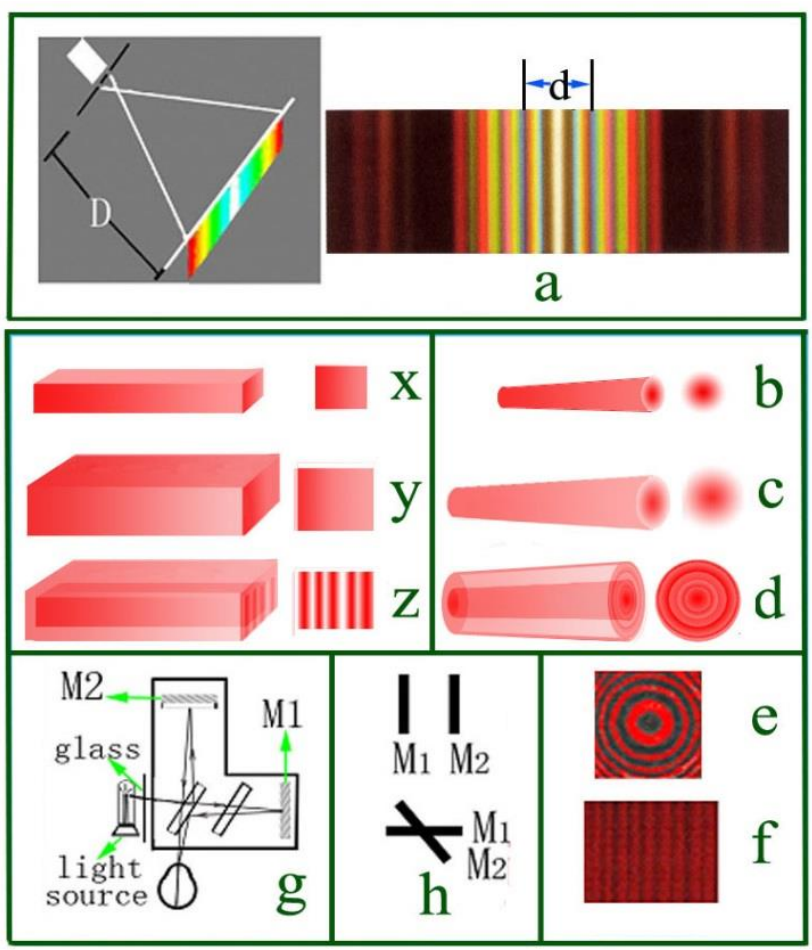

Figure8. Picture $\mathbf{a}$ is the Thomas Young experiment, and picture $\mathbf{g}$ is the A. A. Michelson experiment

The Thomas young experiment etc. ${ }^{[1,2,3,4,5,5,6]}$ were the experiments in order to test the wave theory of light. In the Thomas Young experiment, after light have passed two pinholes, the original status of distributed force in the space has been changed; meanwhile it also produced divergence speed. Thus the two light beams that have passed the two pinholes did not remain original status. For new balance of interaction force between two light beams, the two light beams create interference fringes like above experiment 2. Other details refer to above experiment 2, and 11. In the Michelson experiment, because light beam has divergence velocity when it goes forward, its intensity will be difference along with passed different distance. When M1 is parallel to M2, their status like picture $\mathbf{h}$ in Figure8, their intensity distribution is shown in picture $\mathbf{b}, \mathbf{c}, \mathbf{d}$ of Figure8. The inside light beam has passed shorter distance, so it has higher intensity, like picture b of Figure8, while the outside beam has passed longer distance, so it has lower intensity. Like picture $\mathbf{c}$ of Figure8. This makes their interaction force unbalanced when two light beam encounter. This makes it possible for two light beams to develop circle interference fringes, this shown in picture d of Figure8. The experimental results are showed in picture $\mathbf{e}$ in Figure8. When there is a angle between M1 and M2, their status like picture $\mathbf{h}$ in Figure8, their intensity distribution is shown in picture $\mathbf{x}, \mathbf{y}, \mathbf{z}$ and the interference pattern is shown in picture $\mathbf{f}$ of Figure8. When they has passed the same distance, because their intensity is the same and their mutual force is equal, so they do not develop interference fringes.

\section{Conclusion}

From above, I come to the conclusion that the interference fringes are produced by moving photon created gravitation the inference fringes is caused by force which moving photons produced. The essence of light is particle but not particle-wave duality

The more detail see below book: ISBN: 9789881767592,9789881986863 [7],[8]

\section{Acknowledgment}

I express my appreciation sincerely to Yang Zhenfeng who is in the institute of Mathematic, and my friend Shao shengtang, Jiu guoqiang, Wang xuezheng, Xi xinghua , and Dong qianlin, my middle school teacher Zhang linxiang. My young uncle sister Gou susheng. My young brother Zheng guoman, My young sister Zhnegjunfang.They all gave me a lot of spiritual and financial support when I did the research about this paper. I also express my gratitude for my father Zheng heizi, who gave me great love, and that is the driving force of my moving forward.

\section{References}

[1] Isaac Newton Optics or a treatise of the reflections, refractions, inflections and colors of light London 1730.

[2] Christaan Huygen Treatise on light Silvanus P.Thompson 1912 the university of Chicago press 1945.

[3] M.H.Shamos, Great experiment in physics, Holt, Rinehart and Winston, New York, 1960, p. 93.

[4] M.H.Shamos, Great experiment in physics, Holt, Rinehart and Winston, New York, 1960, p. 108.

[5] G.N. Lewis, Nature, 118 (1926) 874.

[6] L.D. Broglie, Nature, 112 (1923) 540.

[7] Zheng sheng ming Mechanism of interaction in moving matter-the origin of gravitation. ISBN 9789881767592. 2008

[8] Zheng sheng ming Nature mechanism-force origin. ISBN 97898819868632012. 